\title{
Family- and population-based designs identify different rare causal variants
}

\author{
Xue Zhang ${ }^{1}$, Hua He ${ }^{1}$, Lili Ding ${ }^{1}$, Tesfaye M Baye ${ }^{2,3}$, Brad G Kurowski ${ }^{3,4}$, Lisa J Martin ${ }^{1,3,5^{*}}$ \\ From Genetic Analysis Workshop 17 \\ Boston, MA, USA. 13-16 October 2010
}

\begin{abstract}
Both family- and population-based samples are used to identify genetic variants associated with phenotypes. Each strategy has demonstrated advantages, but their ability to identify rare variants and genes containing rare variants is unclear. To compare these two study designs in the identification of rare causal variants, we applied various methods to the population- and family-based data simulated by the Genetic Analysis Workshop 17 with knowledge of the simulated model. Our results suggest that different variants can be identified by different study designs. Family-based and population-based study designs can be complementary in the identification of rare causal variants and should be considered in future studies.
\end{abstract}

\section{Background}

Missing heritability is a major challenge in the discovery of genetic variants responsible for complex disease [1]. One possible reason for the missing heritability is that the current genome approaches focus on common rather than rare variation [2]. However, it is increasingly recognized that rare variants may be responsible for complex disease etiology [3,4]. Thus the next generation of gene discovery should focus on identification of rare variants.

Both family-based and population-based samples have been used to identify variants associated with phenotypes. In recent years, population-based association studies have gained favor because increased power may be obtained $[5,6]$. On the other hand, family-based approaches, such as linkage, are optimally positioned to identify rare variants with large effects $[7,8]$. Because each type of design has strengths and limitations, studies have been conducted using both designs simultaneously. Successes have been reported for common variants, in which the same variants were detected by both designs $[9,10]$. However, inconsistency was also observed $[11,12]$.

It is not clear how family- and population-based analyses behave on rare variants. By applying various methods to

\footnotetext{
* Correspondence: Lisa.Martin@cchmc.org

'Divisions of Biostatistics and Epidemiology, Cincinnati Children's Hospital Medical Center, 3333 Burnet Avenue, Cincinnati, OH 45229, USA Full list of author information is available at the end of the article
}

the population- and family-based data simulated by Genetic Analysis Workshop 17 (GAW17), we compared the power of different designs in the identification of rare (minor allele frequency $[\mathrm{MAF}]<0.01$ ) causal variants.

\section{Methods}

Two data sets were analyzed [13]. One consists of 697 unrelated individuals; the other consists of 697 individuals from 8 extended families. Simulated Q1 phenotypes were used. Analyses were adjusted for age, sex, smoking status, and population stratification using principal components analysis.

\section{Gene-level analysis}

We analyzed the family-based data with a two-point linkage analysis using Sequential Oligogenic Linkage Analysis Routines (SOLAR) 4.10, with identity-by-descent (IBD) matrices from fully informative markers provided by GAW17.

For the population-based data, we collapsed singlenucleotide polymorphism (SNP) information on each of the nine Q1 related genes using three methods. The first method was indicator coding, in which genetic information of a particular gene was dichotomized according to the presence or absence of at least one rare nonsynonymous variant. The second method was percent coding, in which genetic information of a particular gene was 
calculated as $S_{i}=r_{i} / n_{i}$, where $n_{i}$ denotes the number of rare variants successfully genotyped for subject $i$ and $r_{i}$ denotes the number of these variants that carry at least one copy of the minor allele. The third method was weighted-sum collapsing. This method assumes an additive allelic effect by recoding the genotypes into 0,1 , or 2 based on the copy number of the minor allele. Then the single-SNP effect for each of the rare nonsynonymous SNPs was examined using univariate regression. For SNPs with significant $(\alpha \leq 0.1)$ negative effect, the genotypes were converted to 2,1 , and 0 from 0,1 , and 2 , respectively. The genetic information of one particular gene was then summarized as the sum of the numeric genotypes of all rare nonsynonymous SNPs on the gene. The gene-Q1 association was then tested using linear regression.

\section{SNP-level analysis}

In the population-based data, single-SNP association with Q1 was tested using linear regression. In the family-based data, single-SNP association was assessed using a measured genotype approach that compared polygenic models with or without each of the SNPs as a covariate [14]. The quantitative transmission disequilibrium test (QTDT) was performed using JMP Genomics 4.

Analyses were performed with knowledge of the simulated model.

\section{Results}

\section{Gene-level analysis}

In the family-based data, linkage (LOD $\geq 1$ ) to Q1 phenotype was detected in all Q1-related genes, suggestive linkage (LOD $\geq 2)$ was detected in five genes, and strong linkage (LOD $\geq 3$ ) was detected in only the VEGFA and VEGFC genes (Table 1).

For the 200 simulated population-based data, genes FLT1 and KDR showed high power in all three SNP collapsing methods, followed by the VEGFC and VEGFA genes. Power to detect the ARNT, ELAVL4, HIF1A, FLT4, and HIF3A genes was low (Table 2).

\section{SNP-level analysis}

We tested single-SNP association for the 39 causal SNPs in both the population-based and the family-based data. The highest power was observed for markers C13S522 and C13S523 in the population-based data and for markers C6S2981 and C4S4935 in the family-based data (Additional file 1). Although C6S2981 and C4S4935 are rare in the population, they are enriched in families. For SNPs with similar MAFs in both the population-based and the family-based data (C1S3181, C13S431, C4S1861, C4S1878, and C4S1884), our results showed similar power of identification.

Using the QTDT on family-based data, we also tested SNP association for SNPs on chromosomes 1, 4, 6, and 13. The QTDT showed overall lower power than the measured genotype approach (Additional file 1).

Because single-SNP association and the QTDT are two commonly used analysis methods for population- and family-based data, respectively, we compared the power of SNP identification of these two methods. Among the 32 rare causal SNPs in the population-based data, 3 were identified with greater than $50 \%$ power. Among the 11 rare causal SNPs that showed polymorphism in the family-based data, no true causal SNP was identified with greater than $50 \%$ power. In both analyses, high power was observed in common SNPs.

\section{Discussion and conclusions}

Using data simulated by GAW17, in the current study we compared population-based and family-based designs for their ability to identify rare causal variants, as well as gene-level association. We found that the populationbased and family-based designs can result in the

Table 1 Two-point linkage analysis using family-based data

\begin{tabular}{|c|c|c|c|c|c|c|c|c|}
\hline \multirow[t]{2}{*}{ Gene } & \multicolumn{2}{|c|}{ SNP with highest $\beta$} & \multicolumn{2}{|c|}{ SNP with highest MAF } & \multicolumn{4}{|c|}{ Number of simulation } \\
\hline & SNP & $\beta$ & SNP & MAF & $\operatorname{LOD} \geq 1\left(\alpha=10^{-2}\right)$ & $\operatorname{LOD} \geq 2\left(\alpha=10^{-3}\right)$ & LOD $\geq 3\left(\alpha=10^{-4}\right)$ & $\operatorname{LOD} \geq 4\left(\alpha=10^{-5}\right)$ \\
\hline ARNT & C1S6561 & 0.65721 & C1S6533 & 0.011478 & 1 & 0 & 0 & 0 \\
\hline \multirow[t]{2}{*}{ ELAVL4 } & C1S3181 & 0.76911 & C1S3181 & 0.000717 & 3 & 0 & 0 & 0 \\
\hline & & & C1S3182 & 0.000717 & & & & \\
\hline FLT1 & C13S479 & 0.75946 & C13S523 & 0.066714 & 4 & 0 & 0 & 0 \\
\hline FLT4 & C5S5156 & 0.43010 & C5S5133 & 0.001435 & 10 & 1 & 0 & 0 \\
\hline HIF1A & C14S1729 & 0.28532 & C14S1734 & 0.012195 & 1 & 0 & 0 & 0 \\
\hline \multirow[t]{3}{*}{ HIF3A } & C19S4831 & 0.29287 & C19S4799 & 0.000717 & 43 & 3 & 0 & 0 \\
\hline & & & C19S4815 & 0.000717 & & & & \\
\hline & & & C19S4831 & 0.000717 & & & & \\
\hline$K D R$ & C4S1877 & 1.07706 & C4S1878 & 0.164993 & 48 & 9 & 0 & 0 \\
\hline VEGFA & C6S2981 & 1.20645 & C6S2981 & 0.002152 & 198 & 172 & 110 & 55 \\
\hline VEGFC & C4S4935 & 1.35726 & C4S4935 & 0.000717 & 197 & 167 & 126 & 76 \\
\hline
\end{tabular}

$\alpha$ values associated with LOD scores are calculated as described by Ott [15]. 
Table 2 Gene-phenotype association analysis using population-based data

\begin{tabular}{|c|c|c|c|c|c|c|c|c|c|}
\hline \multirow[t]{2}{*}{ Gene } & \multirow[t]{2}{*}{ Number of SNPs } & \multirow[t]{2}{*}{ Number of causal SNPs } & \multirow[t]{2}{*}{ Causal/total SNPs (\%) } & \multicolumn{3}{|c|}{$\begin{array}{l}\text { Number of simulations with } \\
\text { gene detected at } \alpha=0.01\end{array}$} & \multicolumn{3}{|c|}{$\begin{array}{l}\text { Number of simulations with } \\
\text { gene detected at } \alpha=0.0001\end{array}$} \\
\hline & & & & Binary & Percent & Sum test & Binary & Percent & Sum test \\
\hline ARNT & 8 & 4 & 50 & 2 & 2 & 3 & 0 & 0 & 0 \\
\hline ELAVL4 & 3 & 2 & 67 & 2 & 2 & 2 & 0 & 0 & 0 \\
\hline FLT1 & 17 & 8 & 47 & 111 & 111 & 115 & 19 & 19 & 23 \\
\hline FLT4 & 5 & 2 & 40 & 0 & 0 & 5 & 0 & 0 & 0 \\
\hline HIFIA & 5 & 3 & 60 & 3 & 3 & 2 & 0 & 0 & 0 \\
\hline HIF3A & 6 & 3 & 50 & 0 & 0 & 1 & 0 & 0 & 0 \\
\hline$K D R$ & 9 & 8 & 89 & 98 & 162 & 151 & 17 & 52 & 55 \\
\hline VEGFA & 2 & 1 & 50 & 16 & 16 & 20 & 1 & 1 & 1 \\
\hline VEGFC & 1 & 1 & 100 & 86 & 86 & 86 & 11 & 11 & 11 \\
\hline
\end{tabular}

Only rare (MAF < 0.01) nonsynonymous SNPs were used in the analyses.

identification of different causal variants and genes. Because the same underlying simulated model was used for both the family- and population-based data sets, these results suggest that both of these designs have roles in the discovery of rare variant association.

By comparing the identified and unidentified causal genes (Tables 1 and 2), we found several interesting characteristics. Both population- and family-based analysis identified particular genes most of the time (KDR and FLT1 by population-based data; VEGFA and $V E G F C$ by family-based data). In the family-based data, both $K D R$ and FLT1 have five polymorphic causal variants, whereas VEGFA and VEGFC included only a single causal variant each. Based on the expected performance of linkage, one might expect linkage to work better in genes with multiple variants. However, VEGFA and VEGFC show larger effects $(\beta=1.21$ and 1.36 , respectively); thus the ability to detect the $V E G F$ gene may be more reflective of the effect than of the number of variants. On the other hand, the methods we used to identify gene-Q1 association in the populationbased data rely largely on the probability to capture rare variants; thus a higher power for genes with more rare variants (KDR and $F L T 1)$ is not surprising.

When comparing SNP association and the measured genotype approach, we found that power is related to MAF (Additional file 1). When MAF is similar, these two methods show no difference. On the other hand, these two data sets identify different SNPs. Because similar approaches are used, this difference is likely due to the design. The results suggest that for SNPs that are rare in a population, a family-based design may provide an opportunity to enrich the rare SNPs, thus increasing the power to detect the SNP-phenotype association (e.g., C6S2981 and C4S4935). However, a family-based sample may lack polymorphism by chance. In this case, population sampling may be advantageous (e.g., C4S1877 and C4S1889).
When comparing linkage and association results from the family-based data (Table 1 and Additional file 1), we noticed that FLT4 and HIF3A were identified by linkage, but the causal SNPs on these two genes were either nonpolymorphic or had no power to be identified even at the 0.01 level in the association test. Thus, when analyzing family-based data, linkage analysis may be advantageous in the identification of causal regions by using other genetic variations in the same region.

We also compared the association results at the SNP and gene levels from the population-based data (Table 2 and Additional file 1). It appears that gene-level association is not likely to be detected when SNP-level association is lacking. Collapsing the information of the rare SNPs on one particular gene may not enhance the power or provide additional information, as linkage analysis would.

Taken together, these results suggest that neither the family-based nor the population-based analysis we used is sufficient to identify causal variants of next-generation sequence-level data, especially in the context of rare variants. Given that the family-based design offers a variety of advantages (such as segregation with disease rather than just co-occurrence) that cannot be used for unrelated individuals and that may enrich rare variants, the family-based design may also be valuable for genomewide SNP scanning for novel causal variants. Populationand family-based designs can be complementary and should both be considered in future genome-wide association studies.

\section{Additional material}

Additional file 1: Power of the association test in population and family-based data Power\% is the number of replicates detected divided by the number of replicates analyzed multiplied by 100 . The QTDT was performed with 100 replicates; other analyses were performed with 200 replicates. No result was generated by the QTDT for C1S3181 and C4S1890. SNPs with MAF > 0.01 are shaded. na, not applicable. 


\section{Acknowledgments}

The Genetic Analysis Workshops are supported by National Institutes of Health $(\mathrm{NIH})$ grant R01 GM031575 from the National Institute of General Medical Sciences. We would also like to acknowledge NIH grants R01 NS036695, K24 HL69712, K01 HL103165, K12 HD001097-14, and U19 A1070235.

This article has been published as part of BMC Proceedings Volume 5 Supplement 9, 2011: Genetic Analysis Workshop 17. The full contents of the supplement are available online at http://www.biomedcentral.com/1753$6561 / 5$ ? issue $=$ S9.

\section{Author details}

${ }^{1}$ Divisions of Biostatistics and Epidemiology, Cincinnati Children's Hospital Medical Center, 3333 Burnet Avenue, Cincinnati, OH 45229, USA. ${ }^{2}$ Asthma Research, Cincinnati Children's Hospital Medical Center, 3333 Burnet Avenue, Cincinnati, OH 45229, USA. ${ }^{3}$ Department of Pediatrics, University of Cincinnati School of Medicine, 231 Albert Sabin Way, Cincinnati, OH 45267, USA. ${ }^{4}$ Physical Medicine and Rehabilitation, Cincinnati Children's Hospital Medical Center, 3333 Burnet Avenue, Cincinnati, OH 45229, USA. ${ }^{5}$ Human Genetics, Cincinnati Children's Hospital Medical Center, 3333 Burnet Avenue, Cincinnati, Ohio 45229, USA.

\section{Authors' contributions}

XZ carried out the design of the study, analyses using the family-based data and drafted the manuscript. $\mathrm{HH}$ carried out analyses using the populationbased data. LD, TMB and BGK participated in the discussion, and helped to edit the manuscript. LMM conceived of and oversaw the study. All authors read and approved the final manuscript.

\section{Competing interests}

The authors declare that there are no competing interests.

Published: 29 November 2011

\section{References}

1. Eichler EE, Flint J, Gibson G, Kong A, Leal SM, Moore JH, Nadeau JH: Missing heritability and strategies for finding the underlying causes of complex disease. Nat Rev Genet 2010, 11:446-450.

2. Frazer KA, Ballinger DG, Cox DR, Hinds DA, Stuve LL, Gibbs RA, Belmont JW, Boudreau A, Hardenbol P, Leal SM, et al: A second generation human haplotype map of over 3.1 million SNPs. Nature 2007, 449:851-861.

3. Cirulli ET, Goldstein DB: Uncovering the roles of rare variants in common disease through whole-genome sequencing. Nat Rev Genet 2010, 11:415-425.

4. Schork NJ, Murray SS, Frazer KA, Topol EJ: Common vs. rare allele hypotheses for complex diseases. Curr Opin Genet Dev 2009, 19:212-219.

5. Hintsanen $P$, Sevon $P$, Onkamo $P$, Eronen $L$, Toivonen $H$ : An empirical comparison of case-control and trio based study designs in high throughput association mapping. J Med Genet 2006, 43:617-624.

6. Risch NJ: Searching for genetic determinants in the new millennium. Nature 2000, 405:847-856.

7. Knight S, Uh HW, Martinez M: Summary of contributions to GAW Group 15: family-based samples are useful in identifying common polymorphisms associated with complex traits. Genet Epidemiol 2009, 33(suppl 1):S99-S104.

8. Cui Y, Li G, Li S, Wu R: Designs for linkage analysis and association studies of complex diseases. Meth Mol Biol 2010, 620:219-242.

9. Yang Q, Kathiresan S, Lin JP, Tofler GH, O'Donnell CJ: Genome-wide association and linkage analyses of hemostatic factors and hematological phenotypes in the Framingham Heart Study. BMC Med Genet 2007, 8(suppl 1):S12.

10. Lopez-Leon S, Janssens AC, Tiemeier H, Hofman A, Aulchenko YS, Snijders PJ, Claes S, Oostra BA, van Duijn CM: Angiotensinogen M235T polymorphism and symptoms of depression in a population-based study and a family-based study. Psychiatr Genet 2008, 18:162-166.

11. Guhathakurta S, Sinha S, Ghosh S, Chatterjee A, Ahmed S, Gangopadhyay PK, Usha R: Population-based association study and contrasting linkage disequilibrium pattern reveal genetic association of SLC6A4 with autism in the Indian population from West Bengal. Brain Res 2008, 1240:12-21.

12. Goldberger C, Gourion D, Leroy S, Schurhoff F, Bourdel MC, Leboyer M, Krebs MO: Population-based and family-based association study of 5区
UTR polymorphism of the reelin gene and schizophrenia. Am J Med Genet B Neuropsychiatr Genet 2005, 137B:51-55.

13. Almasy LA, Dyer TD, Peralta JM, Kent JW Jr, Charlesworth JC, Curran JE, Blangero J: Genetic Analysis Workshop 17 mini-exome simulation. BMC Proc 2011, 5(suppl 9):S2.

14. Pollin TI, Tanner K, O'Connell JR, Ott SH, Damcott CM, Shuldiner AR, McLenithan JC, Mitchell BD: Linkage of plasma adiponectin levels to 3q27 explained by association with variation in the APM1 gene. Diabetes 2005, 54:268-274.

15. Ott J: Analysis of Human Genetic Linkage. Baltimore, Johns Hopkins University Press; 1992.

\section{doi:10.1186/1753-6561-5-S9-S36}

Cite this article as: Zhang et al:: Family- and population-based designs identify different rare causal variants. BMC Proceedings 2011 5(Suppl 9): S36.

\section{Submit your next manuscript to BioMed Central and take full advantage of:}

- Convenient online submission

- Thorough peer review

- No space constraints or color figure charges

- Immediate publication on acceptance

- Inclusion in PubMed, CAS, Scopus and Google Scholar

- Research which is freely available for redistribution

Submit your manuscript at www.biomedcentral.com/submit
C Biomed Central 